\title{
Televisión 2.0: las estrategias comunicativas en la Web social
}

\author{
Raquel Vinader Segura \\ CES Felipe II, Universidad Complutense de Madrid \\ rvinader@ajz.ucm.es \\ Elena De La Cuadra De Colmenares \\ CES Felipe II, Universidad Complutense de Madrid \\ ecuadra@ajz.ucm.es
}

Recibido: $27 / 07 / 2012$

Aceptado: 30/10/2012

\begin{abstract}
Resumen
La emergencia de las redes sociales, los espacios más populares de la Web 2.0, ha transformado notablemente el entorno comunicativo. Tras el recelo inicial, las cadenas de televisión las han adoptado como parte de sus estrategias digitales ofreciendo así al espectador una experiencia diferencial. Los profesionales de la información tienen ante sí un nuevo reto que, no sólo pasa por adaptarse a un nuevo lenguaje, sino que debe ser capaz de evaluar la fiabilidad de las informaciones que se vierten en estos nuevos espacios. En esta investigación queremos profundizar en las repercusiones de las redes sociales en el periodismo televisivo. Para ello, analizaremos las estrategias de las televisiones en las redes sociales (en concreto Twitter) durante la emisión de diferentes eventos de gran audiencia.
\end{abstract}

Palabras clave: Web 2.0, Televisión, Fuentes de información, Redes sociales, Twitter.

\section{Television 2.0: Comunicative strategies on the Social Web}

\begin{abstract}
The emergence of social networks, the most popular example of Social Web 2.0, has revolutionized the media environment. Despite the initial fear, broadcasters have included them into their digital strategies. This way they are able to offer the audience a differential experience. Professionals of media are facing a new challenge: learning a new communicative language but also to evaluate the reliability of the information present on social networks. This paper presents a study of repercussions of the social networks in Journalism. We will analyze the broadcasters' strategies on social media (specifically Twitter) for the issuance of events with large audiences.
\end{abstract}

Key words: Web 2.0, Television, Information Sources, Social Networks, Twitter.

\section{Referencia normalizada}

VINADER SEGURA, Raquel y DE LA CUADRA DE COLMENARES, Elena (2012): “Televisión 2.0: las estrategias comunicativas en la Web social". Estudios sobre el mensaje periodístico. Vol. 18, núm. especial noviembre, págs.: 909-918. Madrid, Servicio de Publicaciones de la Universidad Complutense.

Sumario: 1. Introducción. 2. Fuentes y metodología. 3. La Televisión 2.0. 4. Fuentes de información. 5. Twitter y televisión: mensajes complementarios. 6. Conclusiones. 7. Referencias bibliográficas

\section{Introducción}

Si bien Internet revolucionó los medios de comunicación, el cambio más significativo se produce diez años después con la llegada de la denominada Web 2.0 o web social. Este término acuñado en 2004 por Tim O'Reilly hace referencia a una segunda generación de Webs basadas en comunidades de usuarios y una gama especial de servicios, como las redes sociales, los blogs, los wikis o las folcsonomías, que fomentan la colaboración y el intercambio ágil de información entre los usuarios (Ribes, 2007:2). 
El salto tecnológico a la Web 2.0 implica la transición a un entorno dinámico donde el internauta es capaz de generar contenidos que comparte con el resto de usuarios (Abuín, 2011). De este modo, un usuario con nociones mínimas de informática se puede convertir en productor y gestor de contenidos, una faceta que antes estaba reservada para los medios de comunicación.

Estas transformaciones en el espacio comunicativo afectan necesariamente a los profesionales de la información que, no sólo deben desarrollar su actividad en los medios tradicionales, sino que deben adaptarse a las bondades de este nuevo medio caracterizado por una actualización permanente de la información y por la interacción con los internautas, que ahora quieren participar en la producción de este tipo de contenidos.

\section{Fuentes y metodología}

La presente investigación tiene como objetivo principal el análisis de las estrategias de las cadenas de televisión españolas ante las redes sociales. Además del estudio de los flujos de información en este tipo de espacios durante las retransmisiones de eventos de grandes audiencias, nos interesa:

1. Estudiar el impacto de las redes sociales en la construcción y difusión de la información en los medios de comunicación masivos.

2. Evaluar la fiabilidad de la información ofrecida por las redes sociales, que en muchos casos se ha puesto en entredicho.

3. Observar los cambios en las competencias y tareas de los profesionales en el entorno de la web 2.0.

4. Comparar la información ofrecida sobre este tipo de acontecimientos de manera simultánea en estos dos medios.

Para conseguir los objetivos propuestos, hemos considerado que la metodología más adecuada es el estudio del caso. Para su consecución se han seguido las recomendaciones de Robert Yin, (1994:13).Uno de los objetivos de esta técnica de investigación es describir situaciones o hechos concretos, que es uno de los propósitos prioritarios de nuestro trabajo. Teniendo en cuenta los cuatro tipos de diseño diferentes para esta metodología que establece Yin hemos considerado que el más adecuado es el Caso único: Unidad múltiple. De este modo, podremos comparar las diferentes estrategias de las empresas de televisión compaginando el uso de Twitter con la retransmisión en directo de eventos multitudinarios: la boda del Duque de Cambridge, las carreras de Fórmula 1 y la Eurocopa de Fútbol. Todos ellos acontecimientos externos al medio televisivo pero capaces de aglutinar a grandes audiencias en el medio tradicional y los espacios sociales emergentes.

\section{La Televisión 2.0}

Desde su nacimiento, Internet se ha convertido en pocos años en un fenómeno imparable pero con importantes repercusiones para los medios de comunicación. Las empresas de televisión vieron en este nuevo medio un poderoso enemigo, un elemento sustitutivo, que podría robarles un número significativo de espectadores haciéndolas 
perder su posición hegemónica en el mercado. Pero, superada la etapa inicial marcada por el miedo al nuevo medio, Internet se configura como un potente aliado para las cadenas de televisión (Chan-Olmsted \& Ha, 2003).

La implantación de estas características en la estructura clásica de televisión no se ha hecho esperar y los radiodifusores la han adoptado, creando modelos híbridos de televisión e Internet. Más allá de las ventajas tecnológicas que permiten al espectador navegar por la Red desde su televisor, nos referimos a experiencias de televisión que se completan a través de los espacios web de las cadenas. Es la denominada Enhanced $T V$ o televisión mejorada, que recoge la interactividad y la capacidad de personalización para ofrecer al espectador a través de sus páginas web un contenido alternativo y diferencial al ofrecido por los medios tradicionales. Mediante esta televisión 'mejorada' la cadena de televisión busca atraer nuevos espectadores e incrementar la fidelidad de los ya existentes. (Ha Bowling \& Chan-Olmsted, 2001:202).

Pero, la transición a la web 2.0 supone un paso más en el desarrollo de Internet y en el mercado de la radiodifusión. De hecho, podemos hablar de la 'Social TV' "que combina los contenidos televisivos con la directa interacción social" (Montpetit, 2009). Por tanto, esta televisión 2.0 configuraría un nuevo modelo de televisión interactiva en la que las redes sociales pueden consolidarse como una herramienta para promocionar y mejorar las audiencias de los contenidos televisivos, al tiempo que reduce la pasividad de los espectadores, que son capaces de comentar y compartir los programas que están viendo. Este nuevo consumo de televisión mejorará la fidelidad al contenido, hará la televisión más atractiva, frenando la erosión de los ratings. La televisión se convierte así en un medio social e interactivo y creando una nueva experiencia de consumo: la interacción social combinada con el contenido televisivo.

Esta televisión social permite renovar el consumo televisivo creando una nueva experiencia comunicativa. El espectador puede ya disfrutar de su contenido favorito con independencia de la plataforma de distribución, de contenidos extra en la página web o compartir sus opiniones sobre los mismos a través de su red social. La sinergia del medio televisivo con la Red transforma las motivaciones y contextos de usos de ambos medios. Mientras que el espectador televisivo se caracterizaba por una escasa interacción con el contenido, reducida al zapping dentro de una limitada oferta de canales de televisión, el nuevo espectador se configura como un usuario activo que, no sólo consume ambos medios de manera simultánea, sino que se muestra interesado en comentar y compartir el contenido televisivo con el resto de amigos de su red social.

De esta manera, se produce una metamorfosis definitiva en el espectador. El espectador televisivo pasivo, se transforma, gracias a la convergencia de la radiodifusión y Red, un usuario activo: viewser. Este término procedente de la convergencia del espectador (televisión) y el usuario (internet) (Konert, 2003:86). La televisión ya no es la única manera de consumir televisión en el hogar. El ordenador, conectado a la banda ancha, se convierte en una alternativa importante, más abierta y flexible. Éste, conectado a la banda ancha, y a través de las redes sociales puede llevar a la creación de comunidades alrededor de un determinado contenido televisivo. Un cambio que se muestra evidente en el discurso inaugural como presidente de Estados Unidos de Barack Obama. Según CNN, este acontecimiento supuso más de 21 millones de cone- 
xiones de video en streaming a través de su página de la red social lo que puede darnos una dimensión del fenómeno. Los datos aportados por Facebook cifran en un millón y medio de actualizaciones relacionadas con el evento en cuestión: 600.000 comentarios en la página de $\mathrm{CNN}$ en esta red social y 4.000 actualizaciones de estado por minuto durante la retransmisión que se incrementaron hasta 8.500 en el minuto que comenzó el discurso. Estas cifras muestran claramente la dimensión que puede alcanzar a nivel mundial un determinado acontecimiento y las repercusiones sociales del mismo.

De hecho, el efecto de las redes sociales en el consumo de televisión está siendo estudiado. Precisamente se han realizado estudios sobre el efecto de este tipo de redes y, más concretamente Facebook, en el seguimiento de los grandes eventos televisivos del mercado norteamericano (Premios Oscar, NFL, etc.). En estos casos, lejos de perjudicar las cifras de audiencia, se ha observado que el seguimiento por parte del público estadounidense mejora gracias a las posibilidades que ofrecen las redes sociales (Poniewozik, 2010).

De este modo, el espectador se manifiesta favorable a la sinergia de los dos medios, ya que es capaz de estar conectado con otros usuarios a través de la Red mientras que consume en tiempo real un determinado contenido. Es decir, el contenido, y su visionado a través del televisor, se convierten en el eje central de las conversaciones de un individuo con su familia y amigos a través de su red social. De esta manera, los espectadores pueden verse inmersos en una nueva experiencia comunicativa que les permite ver la televisión sin tener que estar aislados en su hogar.

Por todo ello, esta nueva experiencia comunicativa combina los elementos más poderosos de ambos medios: the lean-back experience del entorno televisivo con la lean-forward interaction de la Red, abriendo un nuevo mundo de posibilidades en el consumo audiovisual (Montpetit, 2009). El éxito de una empresa de radiodifusión estará condicionado en gran medida por la capacidad de adaptarse a esta nueva situación y ofrecer a sus espectadores nuevas posibilidades de consumo audiovisual (lineal o no lineal) con independencia de su plataforma de distribución o recepción.

\section{Las Fuentes de información 2.0}

Los roles profesionales en las empresas relacionadas con la comunicación han sufrido cambios drásticos con la llegada de las tecnologías. El periodismo ha evolucionado siempre de la mano de la tecnología, al igual que lo ha hecho la Documentación. De hecho, la historia de la Documentación Informativa, que aúna estas dos disciplinas, es la historia de la evolución tecnológica.

Varios estudiosos han analizado la evolución de la documentación en prensa, y coinciden, como señala Cinta Delgado (García Gutiérrez, 1999), en dividir esta evolución en cuatro etapas: una primera (años 60), caracterizada por el uso del propio periódico; una segunda etapa caracterizada por la microfilmación y el ahorro de espacio (años 70); una tercera etapa en la que comienzan a introducirse los ordenadores y las bases de datos (años 80); y la cuarta etapa, en la que se generaliza el uso de las bases de datos a texto completo y el acceso a las redes. Así, vemos cómo la evolución de la documentación en medios de comunicación está estrechamente ligada a las tecnologías. 
Cada avance tecnológico ha introducido un cambio en las tareas profesionales de documentalistas y periodistas; pero estos cambios podrían ser considerados (tomando prestada la nomenclatura de la Documentación) como cambios "formales" en sus quehaceres, no cambios "de contenido" en sus responsabilidades ni en las bases de profesión.

Como advierten Micó, Masip y García-Avilés (2009), "Gracias fundamentalmente a internet, $[. .$.$] los periodistas han adquirido mayor protagonismo en los procesos de$ búsqueda y recuperación de la información, llegando a modificar sus rutinas y su relación con los documentalistas".

Pero los cambios en las profesiones implicadas en el proceso informativo -documentalistas y periodistas- no ocurren en una sola dirección. Como afirman Rintala y Suolanen (2005), "Los cambios en los roles profesionales pueden suceder de diferentes modos: con una transferencia de tareas de un trabajo a otro, con la fusión de uno o más trabajos, o con la suma de trabajos nuevos a otros ya tradicionales"'. Así, observamos que no solo el periodista se convierte en documentalista, buscando información on-line, sino que, en ocasiones, el documentalista se convierte en periodista, publicando en las redes sociales la información con gran rapidez. Ambos roles profesionales deben ser conscientes de los riesgos que entrañan estas prácticas: quizá un periodista no esté consultando las fuentes más recomendables y, por otro lado, quizá el documentalista no deba precipitarse al dar una información sin elaborar.

Internet, sin embargo, sí ha introducido cambios en el trabajo de periodistas y documentalistas: las fuentes de información ya no son un oscuro laberinto de personas o instituciones autorizadas a las que era complejo llegar. Hoy, cualquier persona con un teléfono móvil es una fuente de información. Ni siquiera hace falta que sea un smartphone o teléfono inteligente, basta con tener una línea telefónica a mano para, desde el lugar en que algo acontece, poder narrarlo a la emisora que lo quiera oír.

Las redes sociales han añadido otro cambio que va más allá de la tecnología: la capacidad de que cualquier persona se convierta en emisor de información. Este concepto "cualquier persona", que se expresa en la red con el sufijo "wiki", ha dado como resultado productos como la famosa "Wikipedia". Así, tanto las "wiki" como las redes sociales ofrecen contenidos aportados por cualquier persona. Esto no es necesariamente positivo, como han señalado numerosos autores. No queremos extendernos en este escrito, pero quizá los términos "sabiduría" y "democracia" no combinen del todo bien.

La rapidez con que Twitter permite enviar mensajes nos convierte a todos en periodistas y documentalistas, sin conocer el oficio de ninguna de estas dos profesiones. Como señala Galdón (2002), "hoy más que nunca la labor de documentar y de documentarse es particularmente necesaria si no queremos sucumbir ante la infopolución de informaciones".

1 "Changes in job descriptions can occur in different ways: by transferring tasks from one job description to another, by fusing two or more job descriptions or by adding new tasks to the traditional job descriptions". 
Este autor desarrolla las que considera funciones específicas de la documentación, que alcanza un número total de nueve. Para el propósito de este escrito nos fijaremos en la función critico-verificadora, según la cual, afirma el autor, "es necesario verificar la información procedente de los otros, no solamente con una comprobación fáctica, sino también crítica". Afirma Galdón que la documentación debe comprobar si la información que nos llega es cierta y, aún si lo es, indagar por si tiene algún sesgo e intentar contextualizarla.

Ante el peligro de las informaciones falsas y de la diseminación de rumores, como hemos visto arriba, el usuario de Twitter debe acostumbrarse a buscar las informaciones más veraces posibles, y para ello basta con seguir las cuentas oficiales de personajes, medios o instituciones. En el caso de que el usuario tenga dudas, el símbolo nos asegura que es una cuenta oficial.

Especialmente en eventos programados, como el estudio que nos ocupa, la cantidad de información autorizada y veraz que circula en las redes es apabullante: suficiente para tener los datos al minuto y de la mejor fuente posible. Así, aunque la característica principal de las redes 2.0, como vimos al principio, es que se enriquecen con las aportaciones de todos los usuarios, queremos, con estas líneas, llamar la atención sobre la vuelta a las fuentes autorizadas, esta vez camufladas entre todo tipo de usuarios. Podríamos llamarlo "Redes 2.O” (sí, es una letra "o" mayúscula), dado que remite a la información oficial.

\section{Twitter y televisión: mensajes complementarios.}

Las cadenas de televisión se han dado cuenta de la importancia de las redes y de que el modo de ver televisión ha cambiado con la llegada de las tecnologías portátiles. El consumo televisivo ha sufrido diferentes transformaciones: en ocasiones es una fragmentación: el usuario ve su programa de televisión favorito con independencia de su emisión a través de la web oficial de la cadena a la hora que le es más cómodo. De hecho, según la AIMC, el 12,8\% de los espectadores ve la televisión en diferido aprovechando las mejoras de esta nueva televisión mejorada. Mientras que, en otras ocasiones es una suma: el usuario ve la televisión mientras participa en las redes sociales. Así, las redes sociales se configuran como un nuevo espacio para la puesta en común de contenidos televisivos: el 65\% de espectadores ha comentado en alguna ocasión lo que está viendo en televisión (The Cocktail Analysis, 2011).

Solo a modo de ejemplo, vamos a referir en este escrito algunos casos de esa "suma", en la que las redes sociales, en este caso Twitter, refuerzan los contenidos televisivos con mensajes sobre lo que ocurre en la pantalla. Para ello, hemos seleccionado cuatro casos muy diferentes entre sí: la pero que tienen en común que son retransmisiones de acontecimientos globales capaces de aglutinar audiencias masivas y que han desplegado acciones simultáneas en esta red social:

Uno de los ejemplos más significativos es la boda del ya Duque de Cambridge, primogénito del Príncipe de Gales, con Catherine Middelton en abril de 2011. Durante las horas en que se celebró el enlace, la casa oficial del Príncipe de Gales, y por lo tanto de los Duques de Cambridge, publicaba información de primera mano a través de su cuenta@ClarenceHouse. Nunca antes un periodista retransmitiendo un evento de este 
tipo en directo había tenido tanta información a su alcance. Por ejemplo: el vestido de la novia:

"Miss Catherine Middleton's Wedding Dress has been designed by Sarah Burton at Alexander McQueen".

O el uniforme del Príncipe:

"His Royal Highness The Prince of Wales is wearing the Royal Navy Number One Dress (Ceremonial) to the wedding of Prince William and Miss Catherine Middleton. The Prince of Wales has held the rank of Admiral of the Royal Navy since 2006".

Otro ejemplo de los tuits publicados por Clarence House, encontramos uno con el texto de la oración escrita por los novios (a las 12:30), o el texto íntegro del sermón del obispo (a las 12:45), o, por ejemplo, uno en el que nos facilita un enlace a iTunes con la música de la boda (publicado a las 13:05).

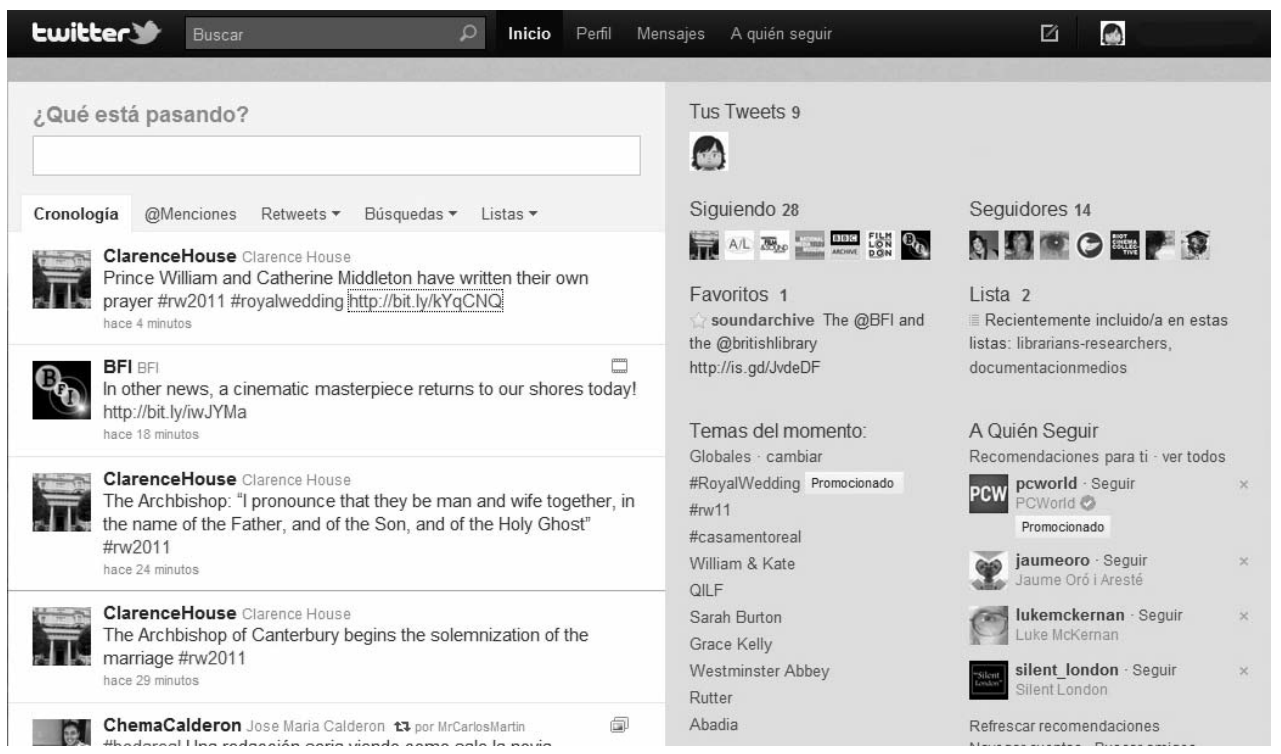

Ilustración 1: Clarence House en Twitter. Fuente: Twitter

También queremos destacar la utilización que se hace de esta red social para fortalecer las relaciones y ampliar la información de los adicionados a la Fórmula 1. Cada fin de semana, las cuentas oficiales de cada una de las escuderías informan minuto a minuto de todo lo que ocurre. Durante la carrera del domingo, se suma la web oficial F1.com, en la que se puede tener toda la información técnica de lo que ocurre en la pista. 


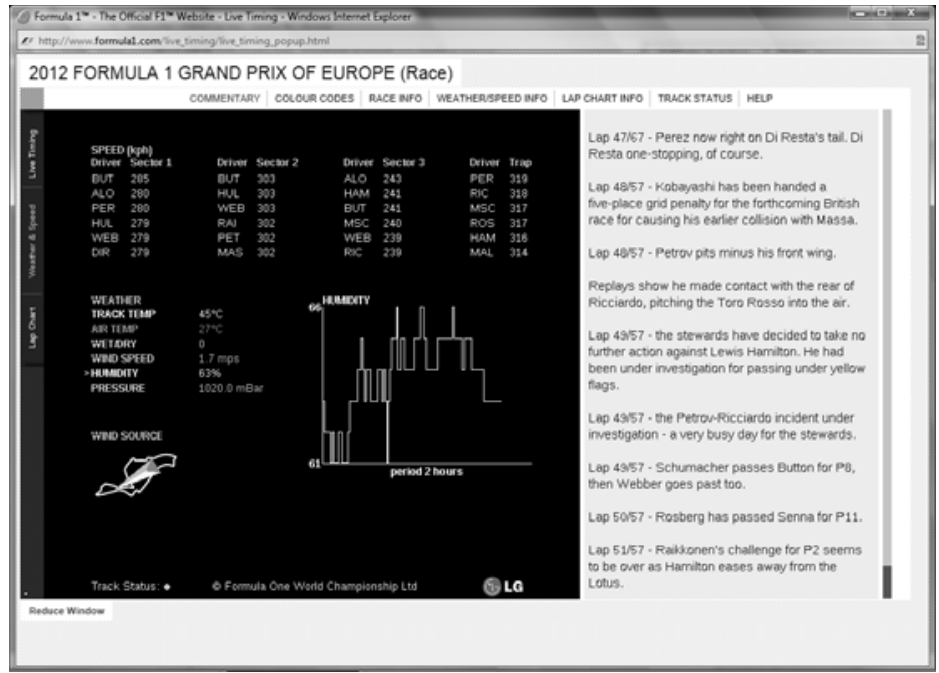

Ilustración 2: Condiciones de la pista durante la carrera. Fuente: www.f1.com

Por último, no podemos olvidar un acontecimiento reciente con gran repercusión en nuestro país: la Eurocopa de Fútbol de 2012. Durante este evento, retransmitido en exclusiva por Mediaset España, se lanzó una estrategia cruzada en redes sociales: mediante un "hashtag" que se alimentaba de contenidos y comentarios a lo largo de la retransmisión de los partidos: \#miteleconlaroja. Pero, no solo era una etiqueta común -cosa que facilita las búsquedas a los usuarios, y el recuento para la medición de audiencias-, sino que, además, Mediaset contrató a cinco personajes famosos para que animaran las retransmisiones con sus comentarios: José Mota, Risto Mejide, José Corbacho, Santiago Segura y David Bisbal.

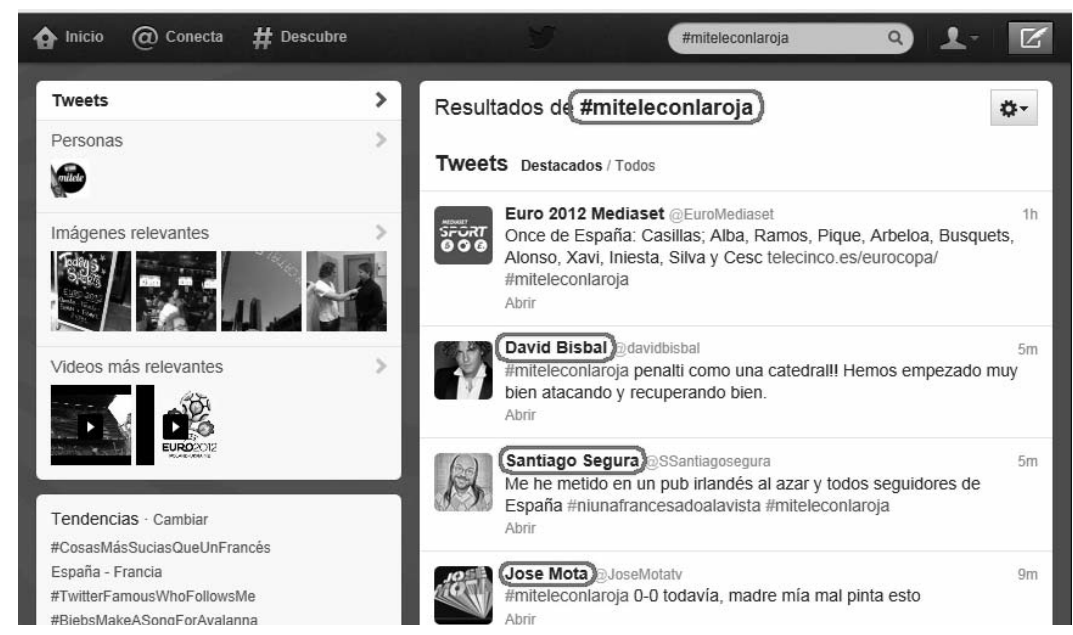

Ilustración 3: Comentarios del hashtag \#miteleconlaroja. Fuente: Twitter 


\section{Conclusiones}

Las redes sociales han modificado significativamente el panorama mediático. En este nuevo espacio de comunicación cualquier persona convertirse en emisor de información. Esto es un arma de doble filo: aunque permite recibir información de lugares antes imposibles, los rumores o las noticias sin contrastar fluyen con rapidez.

Por ello, aparecen nuevos retos para los profesionales de la información. El incremento de las tecnologías de la información, la ingente cantidad de documentos y de informaciones diversas han hecho que tanto los periodistas como los documentalistas vieran su trabajo simplificado pero, al mismo tiempo, se hace cada vez más difícil diferenciar las informaciones veraces. Las redes sociales pueden ser unas fuentes de información valiosísimas pero los periodistas deben evaluar su fiabilidad.

Las redes 2.0 deben convertirse, para los profesionales de la información, en redes 2.O: volver a la fuente oficial, veraz y autorizada, destacada entre voluntarios de buenas intenciones y teclado rápido, para poder percibir, al mismo tiempo, el pulso de la información entre los usuarios anónimos y la información fiable.

\section{Referencias bibliográficas}

ABUÍN, Natalia y VINADER, Raquel (2011): "El desarrollo de la World Wide Web en España: Una aproximación teórica desde sus orígenes hasta su transformación en un medio semántico", en Razón y Palabra, $\mathrm{n}^{\circ}$ 75: www.razonypalabra.org.mx/N/N75/varia_75/varia3parte/31_Avuin_V75.pdf.

AIMC (2012): “La Televisión: Tradicional vs Online', el estudio de AIMC que muestra el comportamiento diario de los telespectadores tanto en el soporte tradicional como en el online". 1 de marzo: www.aimc.es/La-Television-Tradicionalvs, 1050. html.

ANDERSON, Kevin (2009): "Facebook meets TV, literally", The Guardian, 21 January: http://www.guardian.co.uk/technology/blog/2009/jan/21/barackobama-television.

CHAN-OLMSTED, Sylvia y HA, Louisa. (2003): "Internet business models for broadcasters: how television stations perceive and integrate the Internet". Journal of Broadcasting \& Electronic Media, December. New York, Routledge

GALDÓN, Gabriel (coord., 2002): Teoría y práctica de la documentación informativa. Barcelona, Ariel

GARCÍA GUTIÉRREZ, Antonio (ed., 1999): Introducción a la documentación informativa y periodística. Sevilla, MAD

HA BOWLING, Louisa y CHAN-OLMSTED, Sylvia (2001): "Enhanced TV as brand extension: TV viewers' perception of enhanced TV features and TV Commerce on broadcast networks' web sites". The International Journal on Media Management, Vol. 3, n. IV. St. Gallen, Taylor and Francis Group, pp.

KANTAR MEDIA (2011): “La boda de los príncipes Guillermo y Catalina genera un impacto publicitario de más de 46,5 millones de euros". Kantar Media: www.kantarmedia1.es/noticias/view/26 
KONERT, Bertram (2003): "Broadcasters' engagement: from being present to becoming successful, en NOAM, Eli; GOEBEL, Jo; y GERBARG, Darcy: Internet Television. New Jersey, Lawrence Earlbaum, pp. 81-103

MICÓ SANZ, Josep-Lluís; MASIP MASIP, Pere; y GARCÍA-AVILÉS, José Alberto (2009): "Periodistas que ejercen de documentalistas (¿y viceversa?). Nuevas relaciones entre la redacción y el archivo tras la digitalización de los medios". El profesional de la información. Barcelona, Swets \& Zeitlinger

MONTPETIT, Marie-Jose (2009): "Your content, your networks, your devices: Social networks meet your TV Experience". ACM Computers in Entertainment, Vol. 7, $\mathrm{n}$. 3 , Article 34, septiembre

PONIEWOZIK, James (2010): "The World Wide Living Room". The Time. www.time.com

RIBES, Xavier (2007): "La Web 2.0, el valor de los metadatos y de la inteligencia colectiva". Telos, núm. 73. Madrid, Fundación Telefónica

RINTALA, NIINA, SUOLANEN, SANNA (2005): "The implications of digitalization on job descriptions, competencies and the quality of working life". Nordicom Review. Göteborg, Göteborgs Universitet

THE COCKTAIL ANALYSIS (2011): “Televidente 2.0 2011: Tablets, Televisión conectada y redes sociales enriquecen el escenario de consumo de televisión". http://tcanalysis.com/blog/posts/televidente-20-2011-tablets-television-conectaday-redes-sociales-enriquecen-el-escenario-de-consumo-de-television.

YIN, Robert (1994): Case study research: Design and methods, Thousand Oaks, Sage Publishing.

\section{Raquel VINADER SEGURA}

CES Felipe II, Universidad Complutense de Madrid rvinader@ajz.ucm.es

\section{Elena DE LA CUADRA DE COLMENARES}

CES Felipe II, Universidad Complutense de Madrid ecuadra@ajz.ucm.es 